12

\title{
Профилометр для электронного пучка в высоковольтной системе электронного охлаждения синхротрона COSY
}

\author{
() М.И. Брызгунов, ${ }^{1}$ А.П. Денисов, ${ }^{1}$, В.М. Панасюк, ${ }^{1}$, В.В. Пархомчукк, ${ }^{1}$, В.Б. Рева, ${ }^{1,2}$, В.А. Чекавинский ${ }^{1}$ \\ ${ }^{1}$ Институт ядерной фризики им. Г.И. Будкера, \\ 630090 Новосибирск, Россия \\ ${ }^{2}$ Новосибирский государственный университет, \\ 630090 Новосибирск, Россия \\ e-mail: M.I.Bryzgunov@inp.nsk.su
}

Поступило в Редакцию 10 сентября 2020 г.

В окончательной редакции 4 декабря 2020 г.

Принято к публикации 4 декабря 2020 г.

Одной из особенностей высоковольтной электронного охлаждения синхротрона COSY(Германия) является наличие профилометра, предназначенного для измерения распределения плотности тока в электронном пучке. Описана конструкция, методика измерения и результаты измерений профиля электронного пучка в различных режимах работы электронной пушки.

Ключевые слова: ускорители заряженных частиц, электронное охлаждение, диагностика пучков заряженных частиц, профилометры.

DOI: $10.21883 /$ JTF.2021.05.50700.264-20

\section{Введение}

Метод электронного охлаждения, предложенный Г.И. Будкером в 1966 г., позволяет эффективно уменьшать фазовый объем пучков ионов в ускорителях заряженных частиц. В настоящий момент данный метод активно используется во многих ускорительных комплексах, где существует необходимость уменьшить эмиттанс ионных пучков для накопления интенсивности при инжекции или для проведения экспериментов с высоким разрешением по энергии [1].

В большинстве установок, где проводились эксперименты по исследованию электронного охлаждения, были обнаружены эффекты, связанные с развитием неустойчивостей в ионном пучке, приводящие к нагреву пучка и потерям ионов [2]. Кроме того, сильное охлаждение способствует увеличению темпов рекомбинации ионов с электронами.

Чтобы бороться с данными эффектами, было предложено охлаждать ионы пучком электронов с неоднородным профилем пучка, таким, чтобы плотность тока в центре была много меньше плотности на краю (полый пучок). В таком случае можно добиться, чтобы ионы с большими амплитудами бетатронных колебаний охлаждались одновременно с более холодными ионами в центре, тем самым предотвращая образование переохлажденного ядра и позволяя избежать развитие когерентной неустойчивости. Кроме того, поскольку плотность тока в центре низкая, уменьшается скорость рекомбинации. С этой целью в ИЯФ СО РАН была разработана электронная пушка с изменяемым профилем пучка для новых систем электронного охлаждения. Эксперименты, проведенные на специальном испытательном стенде, показали возможность эффективно изменять профиль пучка в широком диапазоне (от узкого параболического распределения, до полого пучка) [3].

Подобная пушка была установлена на высоковольтной системе электронного охлаждения синхротрона COSY [4]. В данной системе электроны двигаются в сильном продольном магнитном поле вдоль всей своей траектории от пушки до коллектора. Наличие неоднородного дрейфового движения в поворотных магнитах, относительно большая длина транспортных каналов, а также относительно большое количество самих поворотов в системе (6 поворотов на $90^{\circ}$ и 2 поворота на $45^{\circ}$ ), приводит к тому, что профиль электронного пучка может существенно искажаться. Таким образом, возникает необходимость контролировать его профиль, для чего в охладителе было решено установить профилометр, позволяющий измерять поперечное распределение плотности тока после секции охлаждения. Выбор типа профилометра для данной системы был осложнен необходимостью обеспечения его работы во всем диапазоне энергий электронов (от $25 \mathrm{keV}$ до $2 \mathrm{MeV}$ ) и необходимостью реализации достаточно компактной конструкции, позволяющей размещать его между катушек продольного магнитного поля.

В качестве возможных решений рассматривались различные методы, использовавшиеся в свое время на аналогичных установках. Например, фосфорный экран, который размещается на пути пучка и регистрирует распределение плотности тока по свечению фосфора [5]. Минусом такого метода является сложность количественного анализа распределения плотности тока в пучке из-за нелинейной связи светимости экрана и тока электронов. Также при работе на больших энергиях фосфорное покрытие будет быстро разрушаться, в связи 
с чем экран придется многократно менять, что в свою очередь, сопряжено с большими техническими неудобствами при работе с вакуумом на уровне $10^{-6} \mathrm{~Pa}$.

Другим методом измерения профиля является тонкая проводящая нить, измеряющая ток электронов, попадающих на нее. Сканируя пучок такой нитью, можно измерить распределение плотности тока. Но это будет только одномерное распределение, т.е. интеграл плотности тока вдоль нити. Два таких профилометра, установленных под $90^{\circ}$ позволяют получить одномерные распределения в обоих направлениях, но для получения двумерного распределения данной информации все еще недостаточно.

В работе [3] вольфрамовая проволока использовалась для измерения профиля электронного пучка по ее свечению. Электроны, попадая на проволоку, теряют энергию, нагревая нить. Подобрав параметры эксперимента таким образом, чтобы нить начала светиться, при этом не разрушаясь, можно измерить свечение нити и после сканирования нитью поперек всего пучка можно восстановить его профиль. Измеряя параллельно с этим ток электронов, попадающих на нить, можно получить полную информацию о распределении плотности тока в пучке. Однако слабая механическая прочность нити ведет к тому, что в процессе эксплуатации ее придется многократно менять, что, как говорилось выше, неприемлемо в данной установке. Поэтому от этого метода решили отказаться.

К неразрушающим методам измерения относится ионизационный профилометр, который измеряет распределение плотности тока, регистрируя распределение вторичных электронов или ионов, образующихся в результате ионизации остаточного газа частицами основного пучка [6]. Для этого на пути пучка создается участок с поперечным электрическим полем, направляющим вторичные электроны или ионы на микроканальную пластину (МКП). На МКП поток частиц усиливается, а затем измеряется с помощью многоанодной пластины либо фосфорного экрана и камеры.

Данный метод обладает многими достоинствами, в связи с чем он все более популярен в настоящее время. Однако дороговизна МКП и необходимость размещения дополнительного оборудования внутри соленоида транспортного канала делают его слишком сложным для данной системы. Кроме того, наличие сильного продольного магнитного поля в канале мешает реализации данного метода, поскольку ионы с низкой энергией, двигаясь поперек магнитного поля, будут поворачиваться за счет силы Лоренца, вследствие чего профиль ионов будет сильно искажаться. Для подавления данного искажения можно увеличивать напряженность поперечного электрического поля. Однако в таком случае напряжения на электростатической системе профилометра становятся слишком большими, чтобы их можно было разместить внутри соленоида транспортного канала.

Данного недостатка лишен метод измерения профиля за счет регистрации фотонов, излучаемых молекулами остаточного газа, после их взаимодействия с пучком [79]. Для этого в определенном месте траектории пучка устанавливается оптическая система с детектором, которая регистрирует свечение остаточного газа. В качестве детекторов используются фотоэлектронные умножители (ФЭУ), либо сборки МКП с фосфорным экраном и камерой. Поскольку регистрируются фотоны, то ни внешние поля, ни поле пространственного заряда пучка не влияют на точность измерения и теоретически такой метод позволяет измерять профиль с очень высоким пространственным разрешением. Недостатком такого метода является очень низкое сечение процесса, которое, как правило, на несколько порядков ниже сечения ионизации. Как следствие, в области профилометра приходится значительно повышать давление остаточного газа, либо создавать струю газа, что сложно сделать в условиях высоковольтного охладителя COSY.

В результате сравнения различных методов было решено изготовить сканирующий профилометр с прямым измерением плотности тока.

\section{1. Конструкция профилометра}

Принцип работы данного профилометра основан на использовании цилиндров Фарадея с электростатическим запиранием вторичных электронов в качестве приемника электронного пучка. Его конструкция показана на рис. 1. Профилометр состоит из следующих основных частей: коллиматор в виде медной пластины с шестью небольшими отверстиями, выделяющий из всего пучка отдельные его участки; приемные электроды, отделенные от проводящих частей конструкции керамическими изоляторами и закрепленные напротив отверстий в коллиматоре; запирающая пластина (также с шестью отверстиями) для подавления вторичной электронной эмиссии из приемных электродов.

В целом можно сказать, что профилометр состоит из шести цилиндров Фарадея, пять из которых расположены горизонтально и один смещен вертикально. Причем приемные электроды всех цилиндров независимы, а коллиматоры и запирающие пластины связанны по потенциалу. Диаметр входных отверстий коллиматора равен $2.5 \mathrm{~mm}$, расстояние между соседними цилиндрами $7 \mathrm{~mm}$. Токовый сигнал с каждого приемного электрода выводится наружу через отдельный герметичный разъем. На запирающую пластину подается отрицательное напряжение $(100-200 \mathrm{~V})$, создающее потенциальный барьер для электронов вторичной эмиссии. Поскольку в спектре электронов вторичной эмиссии есть все энергии от нуля до энергии электронов основного пучка, то данного напряжения недостаточно, чтобы запереть все вторичные электроны. Однако его достаточно, чтобы запереть все истинно вторичные электроны, имеющие энергию до $50 \mathrm{eV}$ и которые составляют существенную часть вторичной эмиссии [10]. Повышение напряжения до величин порядка нескольких киловольт 
не даст существенного прироста в эффективность запирания вторичных электронов, поскольку в этой области спектра сосредоточена малая часть вторичных электронов даже для минимальной энергии электронного пучка в охладителе $(25 \mathrm{kV})$. Чтобы значительно повысить эффективность запирания в данной конструкции профилометра требуется напряжение, сопоставимое с энергией основного пучка, что невозможно реализовать в данной конструкции.

Сам профилометр устанавливается внутри вакуумной камеры выше оси канала таким образом, чтобы не мешать проводке электронного пучка в нормальном режиме работы охладителя.

На рис. 2 показана схема высоковольтной системы электронного охлаждения синхротрона COSY. Электронный пучок генерируется в электронной пушке, расположенной в баке с элегазом 1 , ускоряется в высоковольтной колонне (также расположенной в баке), проходит элементы транспортного канала и попадает в секцию
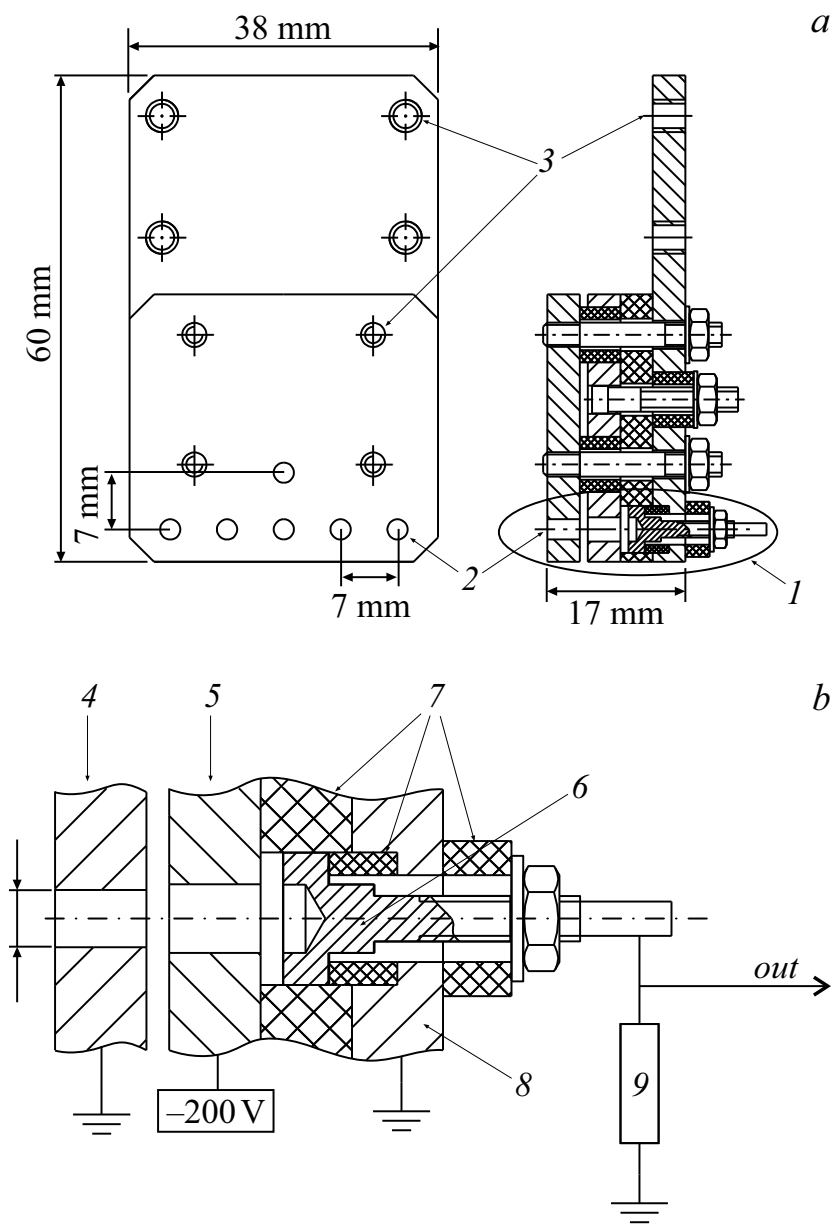

Рис. 1. $a-$ внешний вид профилометра; $b-$ схема профилометра: схема одного цилиндра Фарадея: 1 -- цилиндр Фарадея, 2 - входные отверстия цилиндра Фарадея (всего 6), 3 - крепежные отверстия, 4 - коллиматорная пластина, 5 - запирающая пластина, 6 - приемный электрод, 7 керамические изоляторы, $8-$ несущая пластина, $9-$ измерительный резистор.

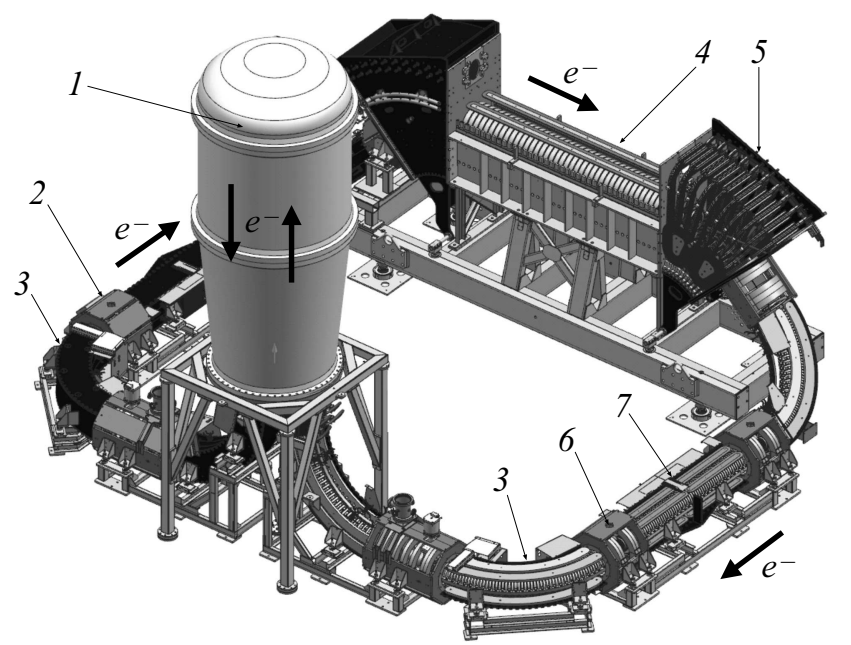

Рис. 2. Схема высоковольтной системы электронного охлаждения синхротрона COSY: 1 - бак с элегазом высокого давления, содержащий электронную пушку, коллектор, ускорительные трубки и систему высоковольтного питания; 2 прямолинейные соленоиды транспортного канала; 3 - поворотные соленоиды транспортного канала; 4 - секция охлаждения; 5 - тороиды; 6 - вставка, в которой расположен профилометр; 7 - прямолинейный соленоид с корректорами для сканирования. Узлы ввода ионного пучка, не изображены на рисунке.

охлаждения 4, где взаимодействует с ионным пучком. Затем пучок возвращается обратно в ускорительную колонну, где он замедляется и поглощается в коллекторе.

Профилометр установлен после секции охлаждения в одном из коротких соленоидов (вставок), предназначенных для стыковки соседних элементов 6 . В вакуумной камере каждой вставки есть боковые фланцы, в которые можно установить дополнительные вакуумные насосы, либо другое оборудование. На один из таких фланцев установлен профилометр.

\section{2. Методика измерений}

Поскольку один цилиндр Фарадея измеряет ток только в одной точке поперечного сечения пучка, для измерения всего профиля необходимо перемещение профилометра относительно пучка (сканирование). С целью упрощения конструкции профилометра было решено не использовать в нем движущихся механизмов, а проводить сканирование самим электронным пучком по поверхности профилометра, отклоняя его за счет катушек поперечного магнитного поля (рис. 3). Корректор вертикального положения пучка в данном прямолинейном участке (7, рис. 2) был усилен (примерно в 2 раза) относительно корректора во втором (симметричном) прямолинейном участке, чтобы его поля было достаточно не только чтобы перемещать пучок в пределах апертуры вакуумной камеры канала, но и чтобы вывести его за апертуру на профилометр. 


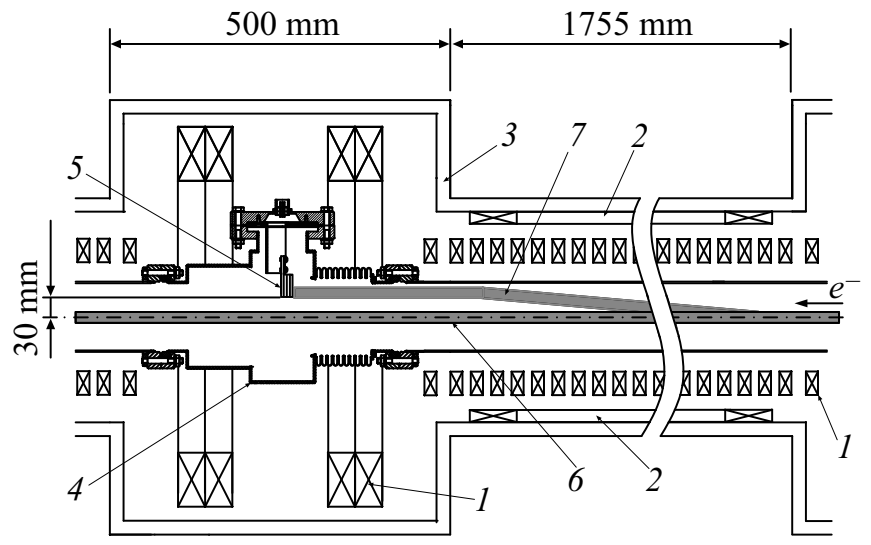

Рис. 3. Схема соленоида с профилометром: 1 - катушки продольного магнитного поля, 2 - корректирующие катушки вертикального поля, 3 - магнитный экран, 4 - вакуумная камера, 5 - профилометр, 6 - траектория электронного пучка в нормальном режиме работы охладителя, 7 - отклоненный электронный пучок при измерении профиля.

Характерное значение продольного магнитного поля в соленоиде $(5-10) \cdot 10^{-2}$ Т. Как правило, во всем транспортном канале делается одинаковым, чтобы избежать возбуждения поперечного движения электронного пучка при прохождении стыков различных элементов. Максимальное значение поперечного магнитного поля от вертикального корректора равно примерно $2 \cdot 10^{-3} \mathrm{~T}$ (ограничено максимальным током источника питания).

Для сканирования в вертикальном направлении ток в катушках вертикального поля изменялся по пилообразному закону (рис. 4). Сканирование в горизонтальном направлении осуществлялось путем изменения тока в горизонтальном корректоре при помощи управляющей программы. Измерение тока с приемных электродов осуществлялось с помощью цифрового осциллографа. Автоматический запуск на осциллографе и длительность одного измерения выставлялись таким образом, чтобы одно измерение соответствовало одному периоду „пилы“ в токе вертикального корректора. После одного измерения данные записывались в файл для дальнейшей обработки. Таким образом, один файл измерений соответствовал одному значению тока в горизонтальном корректоре и содержал в себе всю информацию об одном сканировании в вертикальном направлении. Проведя полное сканирование в горизонтальном направлении, все файлы данных с осциллографа обрабатывались в компьютере для вычисления профиля пучка.

Наличие соседних приемных электродов на заранее известном расстоянии позволяет получить соответствие между током в корректорах и реальным смещением пучка на профилометре. В качестве примера на рис. 4 показаны результаты измерения сигналов с верхнего электрода и центрального электрода в нижнем ряду. Здесь же показана зависимость тока в вертикальном корректоре от времени. Видно, что сигнал в верхнем электроде отстает от сигнала в нижнем на время $\Delta t$, за которое пучок перемещается на профилометре на $7 \mathrm{~mm}$. Именно для калибровки вертикального корректора в профилометре добавлен шестой цилиндр Фарадея, смещенный в вертикальном направлении.

Стоит отметить, что такую калибровку желательно выполнять при каждом новом измерении так как величина смещения зависит не только от тока в корректоре, но и от величины продольного магнитного поля, определяемого основными соленоидами, которое может меняться при изменении режима проводки пучка в охладителе.

Поскольку в горизонтальном направлении профилометр имеет 5 цилиндров Фарадея, можно сократить количество вертикальных сканирований. Для этого необходимо измерять ток со всех цилиндров одновременно и обеспечить сканирование в горизонтальном направлении не на весь диаметр пучка, а только на $7 \mathrm{~mm}$ (расстояние между соседними цилиндрами). Однако при этом необходимо калибровать измерения с каждого цилиндра, поскольку на величину сигнала будут влиять как небольшие конструктивные отличия каждого цилиндра, связанные с погрешностями изготовления и сборки, так и отличия в величине нагрузочного сопротивления на выходе каждого цилиндра.

Мы не стали реализовывать такой способ измерения, а проводили сканирование так, чтобы измерения с третьего (среднего) цилиндра в нижнем ряду содержали в себе
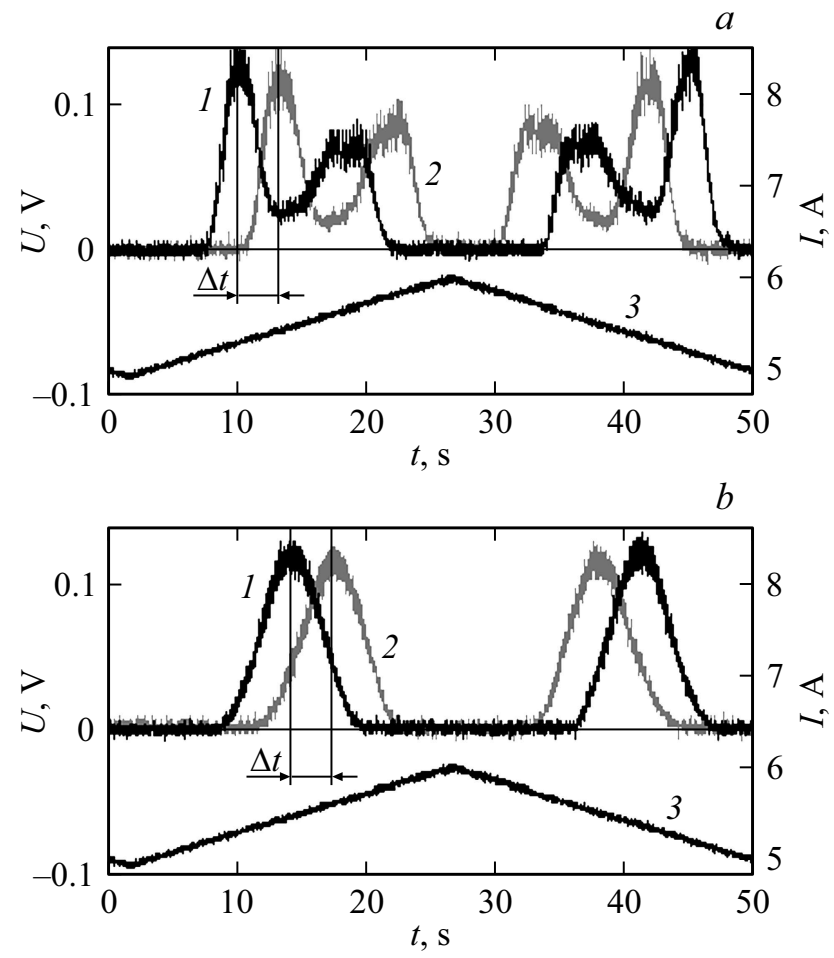

Рис. 4. Результаты измерения сигнала с двух цилиндров Фарадея (слева — для полого пучка, справа — для тонкого пучка) и зависимость тока в вертикальном корректоре от времени: 1 - сигнал с центрального цилиндра в нижнем ряду, 2 - сигнал с верхнего цилиндра, 3 - ток корректора. 
всю информацию о плотности тока по всему сечению сканируемого пучка, т. е. сканирование в горизонтальном направлении перемещало пучок на расстояние большее, чем его диаметр. Таким образом, мы избавились от необходимости калибровать измерения с разных каналов для восстановления распределения плотности тока в пучке. Однако сигнал с соседних цилиндров все равно измеряется для калибровки корректоров (вертикального и горизонтального).

В силу того, что основной высоковольтный источник электронного охладителя не способен выдать большой ток (максимум $1 \mathrm{~mA}$ ), невозможно проводить измерения профиля непрерывного пучка с номинальными токами, необходимыми при охлаждении (сотни $\mathrm{mA}$ ). Чтобы обойти данное ограничение, электроника пушки была модернизирована таким образом, что в режиме измерения профиля напряжение на управляющий электрод подается только на короткий промежуток времени $(1-6 \mu \mathrm{s})$ с периодом 16-1000 ms (оба этих интервала времени можно настраивать). В остальное время на электрод подается отрицательный потенциал (порядка $-2 \mathrm{kV})$, который полностью подавляет эмиссию с катода (запирает пушку). Таким образом, средний ток пучка уменьшается пропорционально скважности сигнала, при этом во время включения пучка пушка работает в номинальном режиме.

Для прямого измерения профиля разрешение профилометра ограничено входным диаметром цилиндра Фарадея $(2.5 \mathrm{~mm})$. Однако теоретически за счет постобработки можно добиться более высокого разрешения. В общем случае для этого необходимо решить интегральное уравнение

$$
J(x, e)=\iint_{R(x, y)} j\left(x^{\prime}, y^{\prime}\right) d y^{\prime} d x^{\prime},
$$

где $J(x, y)$ - измеренное распределение тока в пучке, $j(x, y)$ - искомое распределение плотности тока, область интегрирования $R(x, y)$ соответствует окружности с центром в точке $(x, y)$ и радиусом а равном радиусу входного отверстия цилиндра Фарадея.

Другой более простой способ основан на разбиении пучка в поперечном сечении на тонкие пучки квадратного сечения со стороной, равной требуемому разрешению $\Delta$. Если данный размер $\Delta$ много меньше характерных размеров пучка, то можно считать, что плотность тока в нем постоянна. Рассчитав функцию отклика $g_{i}(x, y)$ (результат измерения профиля i-го пучка профилометром при его единичной плотности тока) для каждого такого пучка и учитывая, что измеренное распределение тока является суперпозицией функций отклика от каждого маленького пучка со своим весом $I_{i}$ :

$$
J(x, y)=\sum_{i} I_{i} g_{i}(x, y),
$$

можно рассчитать распределение плотности тока в пучке, решив уравнение (2) относительно $I_{i}$. Таким образом, задача сводится к решению системы линейных уравнений, для чего можно воспользоваться, например, методом наименьших квадратов.

Стоит отметить, что оба способа очень чувствительны к измерительным шумам, поэтому для повышения разрешения предложенными методами необходимо, прежде всего, повышать точность измерений, уменьшая высокочастотные наводки и добавляя в измерительную цепь фильтры высоких частот.

\section{3. Результаты измерений}

Как уже говорилось, конструкция электронной пушки охладителя COSY позволяет управлять профилем электронного пучка, варьируя соотношение между напряжениями на управляющем электроде и аноде (рис. 5). Подавая на управляющий электрод отрицательный потенциал, подавляется эмиссия с края катода, формируя узкий пучок. Подавая положительный потенциал, наоборот, можно повысить эмиссию с края катода, формируя полый (или трубчатый) пучок. Промежуточные значения позволяют сформировать пучок с практически однородным распределением плотности тока. На рис. 5

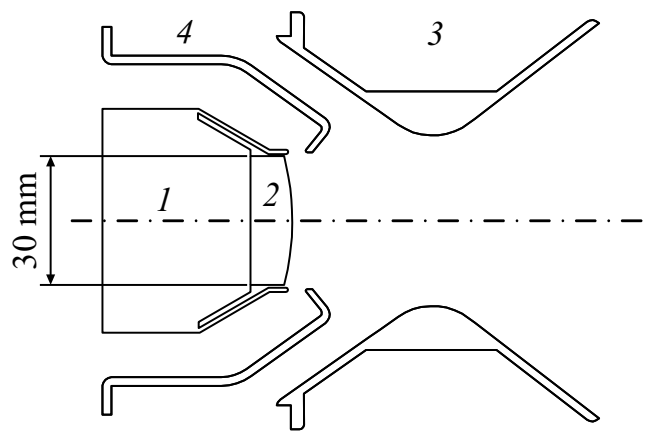

$a$

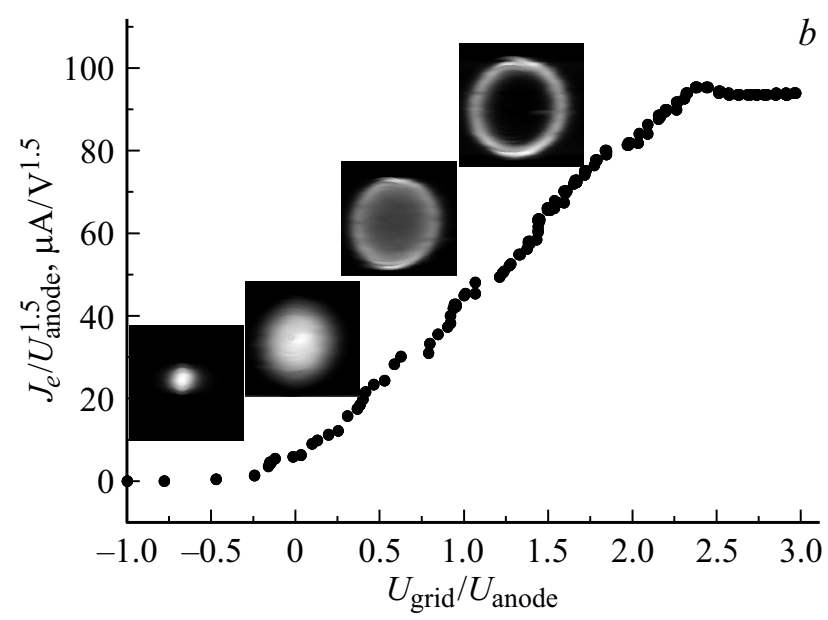

Pис. 5. $a-$ схема электронной пушки: $1-$ катодный узел, 2 - катод, 3 - управляющий электрод, $4-$ анод; $b-$ зависимость первеанса пушки от соотношения напряжений управляющего электрода (,grid“) и анода и характерные профили пучка. 

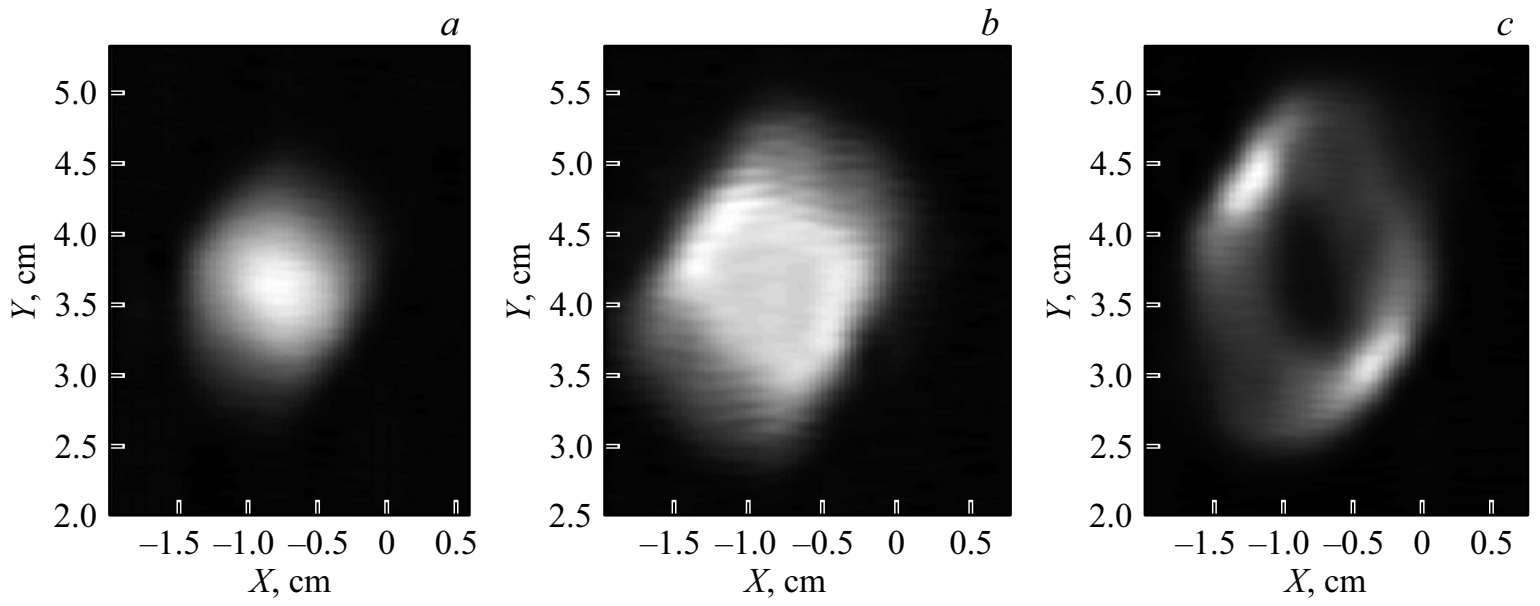

Рис. 6. Результаты измерения профиля пучка при различных режимах работы пушки.

также приведены зависимость первеанса пушки от соотношения напряжений управляющего электрода и анода и характерные профили пучка при некоторых режимах. Данные профили измерялись ранее на специальном испытательном стенде [3]. Результаты этих измерений хорошо согласуются с результатами расчетов оптики пушки [11].

Все измерения профиля, приведенные в настоящей работе, проводились в отсутствие ионного пучка в секции охлаждения на энергии электронов $25 \mathrm{keV}$. Такая энергия была выбрана, поскольку на этом режиме охладитель был настроен лучше всего. Кроме того, для измерений на большой энергии было необходимо настраивать оборудование на дистанционный режим из-за радиации, производимой пучком электронов, погибающем на полной энергии в профилометре. Нами была проведена серия измерений на энергии электронов $300 \mathrm{kV}$, при которой все еще можно было находиться непосредственно вблизи охладителя, однако данные измерения недостаточно полные. Исходя из общих принципов и из полученного опыта, авторы считают, что данный профилометр может работать во всем диапазоне энергии электронов в охладителе (до $2 \mathrm{MeV}$ ).

На рис. 6 показаны результаты измерения профилей пучка в охладителе COSY при помощи описанного выше профилометра, при различных режимах работы электронной пушки.

В целом форма распределения соответствует результатам прежних измерений и расчетов $[3,11]$, однако видно, что пучок имеет не аксиально симметричную форму (хотя катод в пушке круглый). Возможные причины могут быть связаны как с реальным искажением профиля при движении пучка от пушки до профилометра, так и со свойствами самого профилометра.

Искажение в профилометре может происходить изза неоднородности поля вертикального корректора. Поскольку профилометр установлен вдали от оси соленоида, где в нормальном режиме работы пучок проходить

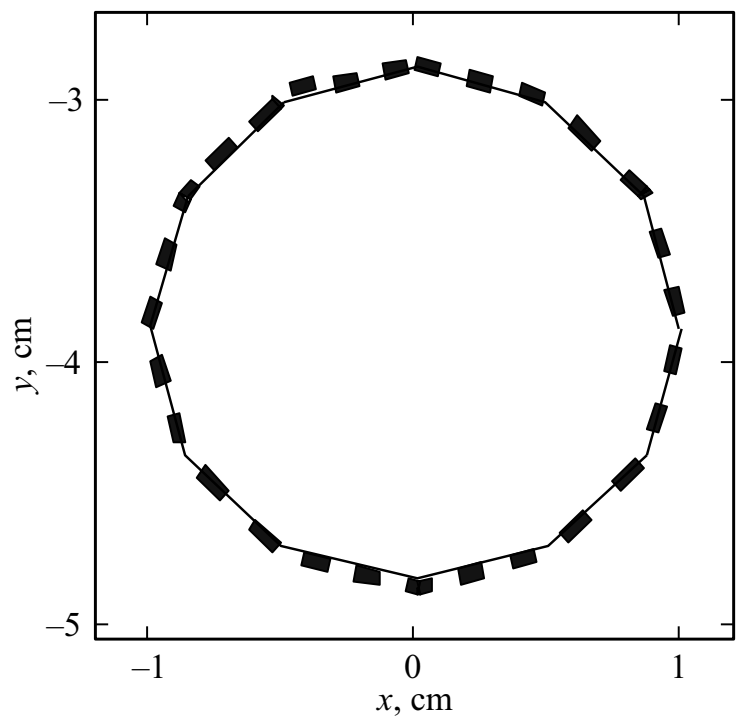

Рис. 7. Результаты расчетов силовых линий магнитного поля в соленоиде. Сплошная линия - начальные точки в начале соленоида (смещенные примерно на $4 \mathrm{~cm}$ для наглядности), штриховая линия - положение силовых линий в области профилометра.

не может, то поле от корректоров в данной области не совсем однородно, вследствие чего электроны в разных частях пучка будут смещаться не одинаково, и профиль будет искажаться.

На рис. 7 приведены результаты расчетов силовых линий магнитного поля при помощи программы MAG3D [12]. Здесь показано как ведет себя набор силовых линий, пущенных в начале (с правого края) соленоида длиной $1755 \mathrm{~mm}$ (рис. 3) с окружности радиусом $1 \mathrm{~cm}$, с центром на оси соленоида. Полное смещение силовой линии от оси соленоида в конце равно примерно $4 \mathrm{~cm}$. Для сравнения на рисунке показана окружность начальных координат силовых линий (смещенная примерно на $4 \mathrm{~cm}$, чтобы совместить центры 
двух окружностей). Видно, что в конце соленоида контур практически совпадает с первоначальной окружностью, что говорит о достаточно хорошей однородности поля корректора. Поскольку на низких энергиях электроны двигаются практически точно по силовым линиям, то можно утверждать, что искажение профиля не связанно с неоднородностью поля корректора.

Искажение профиля пучка при движении от пушки до профилометра может быть связано с наличием поворотов в транспортном канале охладителя.

Для сохранения профиля пучка при прохождении поворота электроны, влетающие в поворот дальше от центра дуги, должны поворачиваться с большим радиусом. Это невозможно сделать при однородном поперечном поле, поэтому поле должно иметь градиент. Рассмотрим, как будет двигаться частица, влетающая в поворот с локальными поперечными координатами $x, y$. Для этого пренебрежем быстрыми ларморовскими осцилляциями, а будем рассматривать только дрейфовую составляющую движения.

Поперечная дрейфовая скорость такого электрона равна

$$
\mathbf{V}=c \frac{\left[\mathbf{F}_{\perp} \times \mathbf{B}\right]}{e B^{2}} .
$$

Считая, что компоненты поперечного магнитного поля равны

$$
\left\{\begin{array}{l}
B_{x}=-B_{0} n \frac{y}{R}, \\
B_{y}=B_{0}\left(1-n \frac{x}{R}\right),
\end{array}\right.
$$

можно записать дрейфовые уравнения движения в линейном приближении:

$$
\left\{\begin{array}{l}
V_{x}=-V_{0} n \frac{B_{0}}{B} n \frac{y}{R}, \\
V_{y}=V_{0} \frac{B_{0}}{B}(1-n) \frac{x}{R} .
\end{array}\right.
$$

Здесь $n-$ показатель спада магнитного поля, $B-$ продольное поле в соленоиде, $B_{0}$-вертикальное (поворотное) магнитное поле на оси, $R-$ радиус поворота, $V_{0}$ - продольная скорость электрона. Из данных уравнений следует, что для сохранения круглой формы пучка электроны должны двигаться вокруг оси по круговой траектории. Это условие можно записать в виде $\frac{V_{x}}{y}=-\frac{V_{y}}{x}$, откуда следует, что необходимо, чтобы показатель спада был равен 0.5 .

Однако, поскольку реальный показатель спада не равен точно 0.5 , то проход пучком даже одного поворота искажает его форму. На пути от пушки до профилометра пучок проходит 4 поворота на $90^{\circ}$ и 2 поворота на $45^{\circ}$ (рис. 3). Вследствие этого профиль может измениться достаточно сильно. Однако расчеты показывают, что на низких энергиях, при которых проводились измерения профиля, влияние данного эффекта очень мало и не может объяснить столь сильное искажение профиля пучка.

Исходя из приведенных выше результатов расчетов (а также расчетов, проведенных при проектировании охладителя), следует, что наблюдаемая асимметрия профилей, скорее всего, связана с искривлением самого пучка при движении от пушки до профилометра и вызвана неточностями изготовления и сборки транспортного канала.

\section{4. Измерение при малых токах пучка}

С технической точки зрения, чтобы обеспечить возможность включать пучок на короткое время за счет управляющего электрода, необходимо существенно усложнить его источник питания. Кроме основного высокого напряжения, к этому же электроду подводится переменное напряжение с амплитудой порядка $10 \mathrm{~V}$ и частотой $3 \mathrm{MHz}$, которое суммируется с основным напряжением для модуляции тока пучка с тем, чтобы иметь возможность измерять его положение в пикапах для транспортировки пучка через систему транспортных каналов и для совмещения его с ионным пучком в секции охлаждения. Такая схема, объединяющая в себе сразу возможность модулировать основной сигнал синусоидой амплитудой $10 \mathrm{~V}$ и полностью запирать пушку за время примерно $1 \mu \mathrm{s}$, получается очень громоздкой, поэтому появилось желание проверить возможность измерения профиля пучка без использования импульсного режима. Были проведены измерения с пучком постоянного тока величиной порядка $100 \mu \mathrm{A}$, для чего на анод и управляющий электрод подавались очень низкие напряжения (порядка $10 \mathrm{~V}$ ). На рис. 8 показаны результаты измерения профиля пучка при таком режиме.

Здесь показаны результаты для тонкого и полого пучка. Видно, что для полого пучка результаты измерения намного более „шумные“, чем на рис. 6. Скорее всего, это связанно с очень низкими напряжениями на электродах пушки. Поскольку источники питания электродов рассчитаны на напряжения в несколько киловольт, их абсолютная стабильность находится на уровне $(0.1-1) \mathrm{V}$, что на рабочем напряжении дает необходимую относительную стабильность $10^{-3}-10^{-4}$. Однако при работе на низких напряжениях электродов (которые
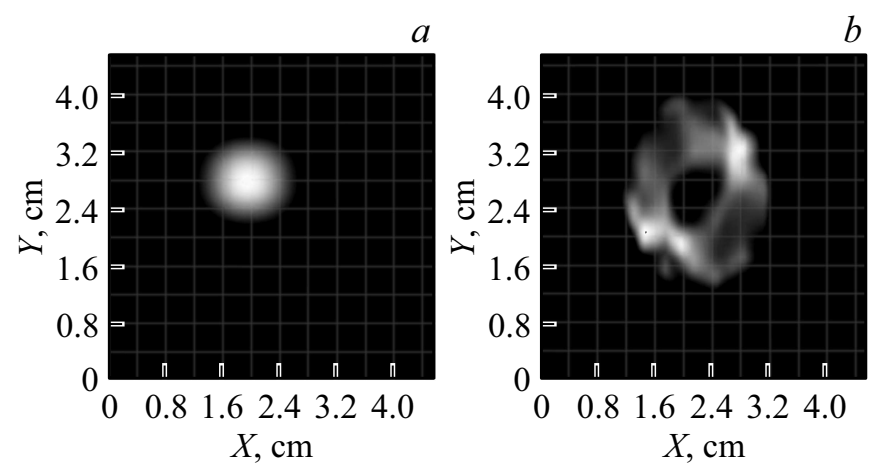

Рис. 8. Результаты измерения профиля пучка без использования импульсного режима работы пушки: $a$ - тонкий пучок; $b-$ полый пучок. 
никогда не требуются при охлаждении), относительная стабильность может достигать уровня $10 \%$ и выше, что и приводит к столь „шумному“ результату.

Для формирования тонкого пучка требуется более высокое напряжение на аноде и большее по модулю (чем в случае с полым пучком) отрицательное напряжение на управляющем электроде. Вследствие чего результаты измерений профиля тонкого пучка не такие „шумные“.

Результаты показывают, что такой способ измерения на малых токах может применяться для измерения профиля описанным профилометром, однако для этого требуется модификация источников питания, чтобы повысить их стабильность. В качестве альтернативы такому режиму измерения можно рассмотреть импульсный режим, где запирание пушки производится не за счет управляющего электрода, а за счет анода. В таком случае для открытия пушки на анод подается номинальное напряжение (порядка нескольких киловольт), а для запирания на него подается отрицательное напряжение. Электроника для такого режима будет значительно проще, поскольку не требуется совмещать запирание пушки и модулирование тока для измерения пикапами. Модулирующий сигнал, как и раньше, будет подаваться на управляющий электрод.

Недостатком такого способа запирания пучка является больший перепад напряжения по сравнения с запиранием при помощи управляющего электрода. Кроме того, не вполне ясно как будет работать пушка, если при запертом аноде на сетке выставлено положительное напряжение. В таком случае эмитируемые с катода электроны могут достичь управляющего электрода, что приведет к десорбции газа с его поверхности и ухудшению вакуумных условий. А это, в свою очередь, может привести к деградации поверхности оксидного катода. Поэтому данный способ реализации импульсного режима требует дополнительных исследований.

\section{Заключение}

Результаты испытаний профилометра на высоковольтной системе электронного охлаждения для синхротрона COSY показали его эффективность и достаточно высокую точность при относительной простоте его конструкции. Данный профилометр не требует дополнительных механических приспособлений для сканирования пучка, поскольку сканирование производится самим пучком при помощи магнитных корректоров поперечного положения пучка.

Поскольку данный профилометр измеряет непосредственно ток пучка, попадающий на приемный электрод цилиндра Фарадея, то результат измерений дает не только форму распределения в относительных единицах, но и реальное распределение плотности тока в пучке. При этом необходимо помнить о том, что пушка работает в импульсном режиме и может корректировать результат измерения, учитывая скважность.
Разрешение такого профилометра определяется размерами входного отверстия цилиндра Фарадея, диаметр которого в данном устройстве равен $2.5 \mathrm{~mm}$. При необходимости измерять более точно можно изменить конструкцию, уменьшив размер отверстия. Кроме того, можно добиться более высокого разрешения за счет постобработки результатов измерений. Однако в этом случае требуется повышать точность измерений, подавляя шумы.

Для увеличения точности также следует уделить внимание однородности поля основного соленоида и корректоров области профилометра. В противном случае искажение профиля из-за неоднородностей поля могут быть выше, чем точность, определяемая диаметром входного отверстия.

Измерения можно проводить на малых токах пучка без использования импульсного режима, однако для этого необходимо повышать стабильность высоковольтных источников питания пушки. В качестве другой альтернативы можно рассмотреть импульсный режим, при котором импульсное напряжение подается не на управляющий электрод пушки, а на анод, что упростит управляющую электронику. Однако данный способ требует дальнейших исследований.

\section{Конфликт интересов}

Авторы заявляют, что у них нет конфликта интересов.

\section{Список литературы}

[1] Н.С. Диканский, И.Н. Мешков, В.В. Пархомчук, А.Н. Скринский. УФН, 188 (5), 481 (2018).

[2] L. Hermansson, D. Reistad. Nucl. Instrum. Methods Phys. Res., A, 441, 140 (2000).

[3] А.В. Бублей, В.М. Панасюк, В.В. Пархомчук, В.Б. Рева. Измерения профиля интенсивного электронного пучка (Препринт ИЯФ 2004-77, Новосибирск, 2004)

[4] A.V. Ivanov, M.I. Bryzgunov, A.V. Bubley, et al.Electron Gun and collector for $2 \mathrm{Mev}$ electron cooler for COSY. Proceed. RuPAC 2010 (Protvino, Russia, 2010), p. 233.

[5] В.В. Смалюк. Диагностика пучков заряжсенных частии, в ускорителях, под ред. Н.С. Диканского (Параллель, Новосибирск, 2009), 294 с.

[6] C. Böhme, J.L. Conradie, J. Dietrich, V. Kamerdzhiev, T. Weis. Beam Profile Monitoring at COSY via Light Emitted by Residual Gas. in Proc. 9th European Workshop on Beam Diagnostics and Instrumentation for Particle Accelerators (DIPAC'09) (Basel, Switzerland, May 2009, paper TUPB10), p. 185.

[7] Р.Г. Бикматов, А.Г. Борискин, В.И. Куделайнен, И.Н. Мешков, В.В. Пархомчук. Атомная энергия, 24 (1), 63 (1968).

[8] G. Burtin, J. Camas, G. Ferioli, R. Jung, J. Koopman, R. Perret, A. Variola, J.M. Vouillot. The luminescence profile monitor of the CERN SPS. Proceed. EPAC 2000 (Vienna, Austria, 2000), p. 256. 
[9] J. Dietrich, C. Boehme, T. Weis, A.H. Botha, J.L. Conradie, P.F. Rohwer. Beam profile measurements based on light radiation of atoms excited by the particle beam Proceed. PAC07 (Albuquerque, New Mexico, USA), p. 3955.

[10] K.A. Wright, J.G. Trump. J. Appl. Phys., 33, 687 (1962).

[11] A. Bubley, A. Goncharov, A. Ivanov, E. Konstantinov, S. Konstantinov, A. Kryuchkov, V. Panasyuk, V. Parkhomchuk, V. Reva, B. Skarbo, B. Smirnov, B. Sukhina, M. Tiunov, M. Zakhvatkin, X. Yang. The electron gun with variable beam profile for optimization of electron cooling. Proceed. EPAC 2002 (Paris, France, 2002), p. 1356.

[12] М.А. Тиунов, Б.М. Фомель. Расчет трехмерных магнитных систем с железом (Препринт ИЯФ 83-150, Новосибирск, 1983) 